\title{
The Incidence of Prostate-Specific Antigen Test in a Country With a Limited Social Perception of Prostate Cancer 2006-2016: Disparities Manifested by Residential Area
}

\author{
Young Hwii Ko', Byung Hoon Kim² \\ 'Department of Urology, College of Medicine, Yeungnam University, Daegu, Korea \\ ${ }^{2}$ Department of Urology, School of Medicine, Keimyung University, Daegu, Korea
}

\begin{abstract}
Purpose: To trace the incidence of serum prostate-specific antigen (PSA) tests by the residential areas of Korea, where social awareness on prostate cancer (PCa) is limited due to the absence of a public screening.

Materials and Methods: From Korean national health insurance data, the number of men older than 40 years who performed PSA testing without PCa or before the enrollment on the national registry during 2006-2016 was identified, then its association with the incidence of PCa were analyzed by a residential area categorized into metropolitan, urban, and rural area.

Results: PSA testing was performed more in the metropolitan area in 2006-2008, but performed the most prevalently in the rural area since 2010. Among the 17 administrative districts, the most PSA-tested district was Jeollabuk-do (9.36\%) in 2016, which was Seoul (2.85\%) in 2006. The mean repetition number of PSA tests during 11 years of the study period was highest among the inhabitant of Seoul (3.25 times, $\mathrm{p}<0.0001$ ), followed by Gwangju (2.98 times), another metropolitan city. The mean repetition number of PSA tests during the study decade was in the order of metropolitan area (2.97 times), rural area (2.83 times), and urban area (2.75 times, $\mathrm{p}<0.0001)$. In every year, however, the incidence of PCa was the highest in a rural area, where had the oldest mean age.

Conclusions: Despite a recent increase of PSA screening and diagnosis of PCa especially in the rural area, the opportunity of repeated testing was presented more to the inhabitant of a metropolitan area.
\end{abstract}

Key Words: Prostate cancer, PSA screening, Incidence
Received December 6, 2021

Revised January 6, 2022

Accepted January 11, 2022

Corresponding author:

Young Hwii Ko

Email: urokyh@naver.com

https://orcid.org/0000-0002-9150-4292

\section{INTRODUCTION}

Because the incidence of prostate cancer $(\mathrm{PCa})$ in Korea currently is led by its spread among the elderly population, it is projected to increase significantly in parallel with the accelerating trend of societal aging, ${ }^{1,2}$ In the detection of PCa, serum prostate-specific antigen (PSA) testing plays a pivotal role, given that the majority of contemporary $\mathrm{PCa}$ does not manifest any specific symptoms besides ambiguous male lower urinary tract symptoms that originated more frequently from the concomitant benign prostate hyperplasia. Therefore, the most serious consequence of current Western-based prohibitive guidelines on the PSA test, particularly for the elderly in Korea, is that their negative 
impact on the establishment of screening policy for the men who may benefit from the PSA test, given this distinctive epidemiologic background on the PCa.

As with other Asian countries, ${ }^{3}$ the incidence of PCa in Korea is rapidly increasing in the last decade. Since 2002, when PCa was firstly reported as the 5th most commonly developing male malignant disease, the incidence of PCa has kept increasing jumping one spot to the 4th from 2016. In the most updated report (2019), PCa became the 3 rd prevalent cancer among the Korean male, and 2nd most common cancer especially in the aged men over 65 years old, ${ }^{4}$ despite a limited social awareness dominantly caused by the absence of a nation-driven public screening policy influenced by contemporary western recommendations.

Given the age-related increasing incidence of PCa along with the national-wide trend of aging which has been manifested more remarkably in a rural than an urban area, we traced the incidence of serum PSA testing by the residential areas of Korea and investigated its impact on the detection of PCa.

\section{MATERIALS AND METHODS}

\section{Data Source and Study Subjects}

Data used in this study were obtained from the National Health Insurance Review and Assessment Service of Korea (NHIS), which covers approximately 98\% of its population and provides universal health coverage. The Korean NHIS database includes almost all medical data, including diagnostic codes, procedures, and outcomes (deaths), and it also includes sociodemographic information such as age, health insurance premiums, and residential area. All personal identification numbers are encrypted before data processing to comply with the privacy guidelines of the Health Insurance Portability and
Accountability Act. All study procedures and ethical aspects were approved by the Yeungnam University Hospital Institutinal Review Board (approval number: YUMC-2019-11-012-002).

From the NHIS data, male subjects older than 40 years who performed the PSA test from 2006 through 2016 were identified. The PSA test code utilized in this study was B5490, C4280, C7428, and the tests performed just before 3 months, and after the registration into NHIS as C61 were excluded. Patients who were newly diagnosed with PCa and registered in the NHIS with an International Statistical Classification of Diseases, 10th revision code of C61, as well as V193/194 each year between the study periods, were also investigated.

\section{Study Design and Endpoints}

Currently, Korea consists of 17 administrative districts categorized into (1) Seoul, the capital city, (2) 6 metropolitan cites including Incheon, Daejeon, Daegu, Gwangju, Ulsan, and Busan, (3) Sejong special self-governing city (a planned city as an administrative capital), (4) 8 provinces including Gyeonggi-do, Gangwon-do, Chungcheongbuk-do, Chungcheongnam-do, Jeollabuk-do, Jeollanamdo, Gyeongsanbuk-do, Gyeongsangnam-do, and (5) Jeju self-governing province. We simply categorized these 17 districts into 3 residential areas based on the current population including (1) metropolitan area (Seoul, 6 metropolitan cities, and Gyeonggi-do province), (2) urban area (all the other administrative cities population more than 50,000), (3) rural area (the rest administrative districts including gun/eup/myeon). Considering a reported association between the incidence of $\mathrm{PCa}$ and aging, the mean age of each residential area was investigated from annual statistics in Korea.

The primary endpoints of this study were (1) to determine the prevalence of PSA test by the residential area, and (2) to identify an association 
between the implementation of PSA test and the newly registered PCa by the residential area, given the reported disparities in the prevalence of $\mathrm{PCa}$, based on age and the socioeconomic status. ${ }^{5}$

\section{Statistical Analysis}

To compare the characteristics and outcomes between each group, Student t-test was used for continuous variables, and the chi-square test was used for binary and categorical variables. Cancer incidence rates were calculated per 1,000 personyears. To determine the association between the PSA test and the development of PCa, correlation analysis was utilized. Two-sided p-values of $<0.05$ were considered to be statistically significant. All statistical analyses were performed using SAS ver. 9.4 software (SAS Institute Inc., Cary, NC, USA).

\section{RESULTS}

\section{The Difference of PSA Incidence by the Residential Area 2006-2016}

The incidence of the PSA test across 3 residential areas was significantly different, which was performed highest in the metropolitan area between 2006 and $2008(\mathrm{p}<0.001)$ (Table 1). Though it was carried out the most prevalently in a rural area since 2010, the incidence of PSA tests was consistently increased across all 3 residential areas without a recession from $2.41 \%-2.56 \%$ in 2006 to $7.20 \%-7.52 \%$ in 2016 (Fig. 1).

A significant difference was also observed in the PSA testing incidence among the 17 administrative districts except for Sejong city (a newly built administrative city), which is about double fold in the most frequently performed district of Jeollabukdo $(9.36 \%)$ than the least frequently performed district of Jeju self-governing province (4.31\%) in 2016 (Fig. 2). The most PSA-tested district was

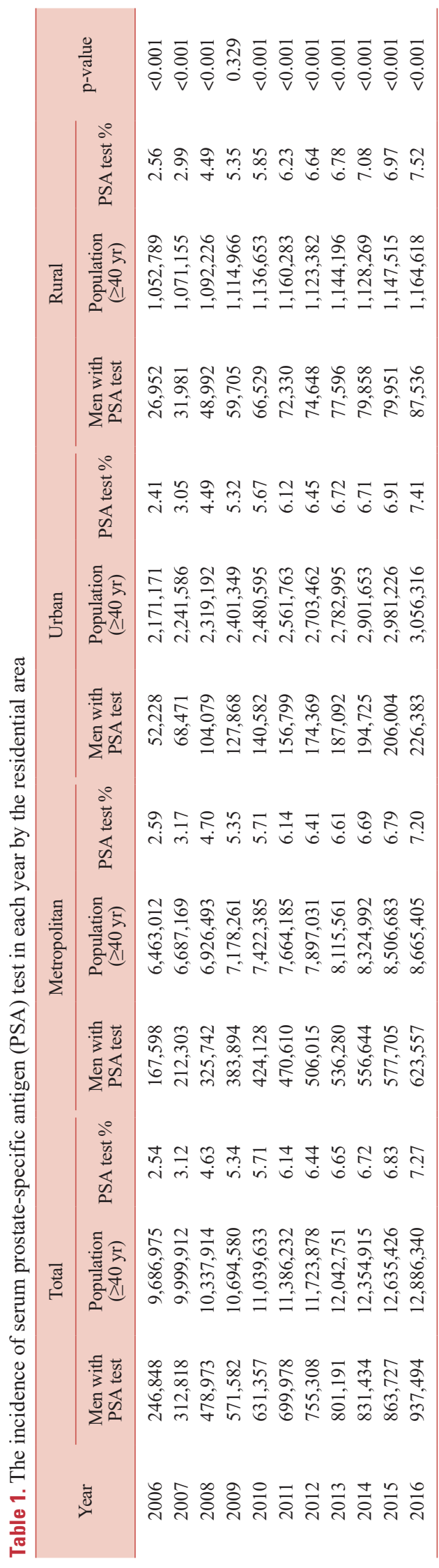




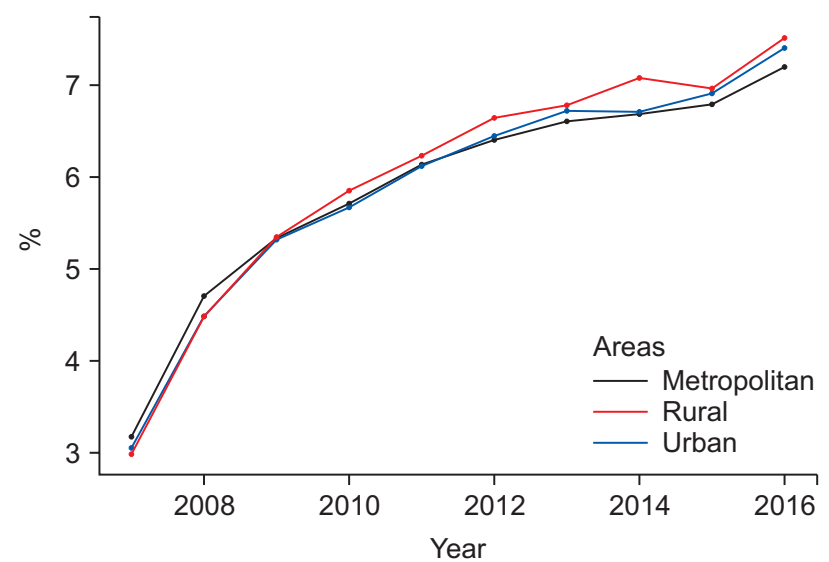

Fig. 1. The incidence of serum prostate-specific antigen test in each year by the residential area.
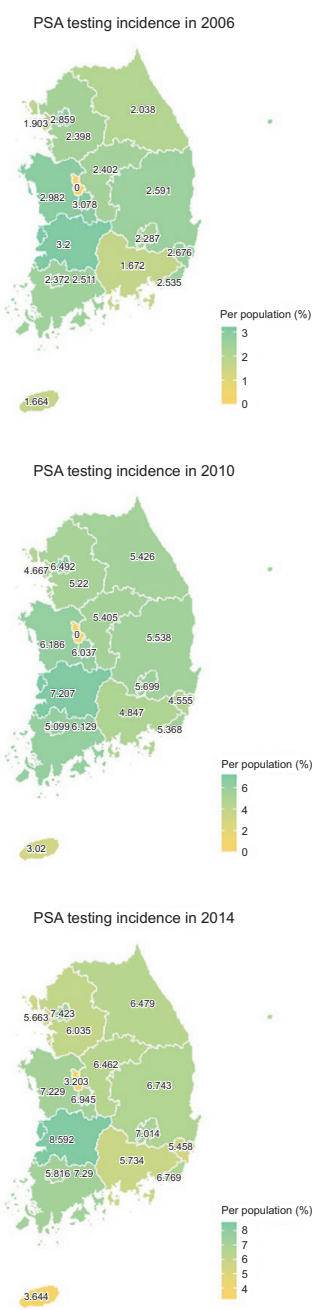

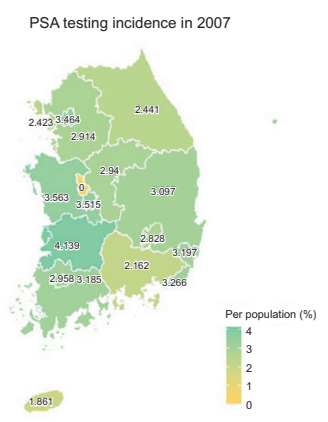

PSA testing incidence in 2011

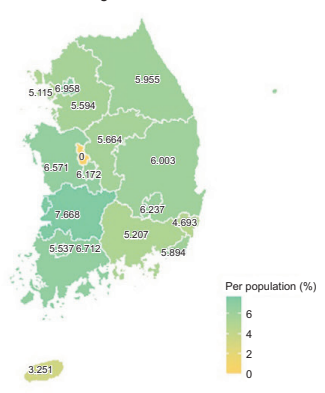

3.251

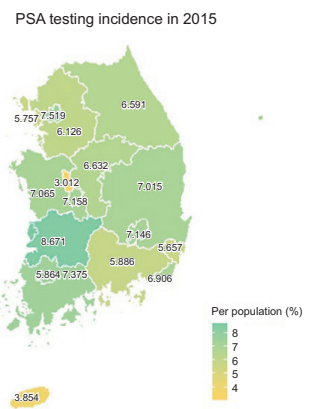

Jeollabuk-do in 2016, which also was Seoul in 2006.

The mean repetition number of PSA tests during 11 years of the study period was highest among the inhabitant of Seoul (3.25 times, $\mathrm{p}<0.0001$ ), where is a sole district more than 3 repeated tests were performed on average. The mean repetition number of PSA tests during the study decade was in the order of metropolitan area (2.97 times), rural area (2.83 times), and urban area (2.75 times, $\mathrm{p}<0.0001)$.

Fig. 2. The incidence of serum prostate-specific antigen test in each year by the 17 administrative districts. 


\section{The Difference of PCa Incidence by the Residential Area 2006-2016}

During the research period, the incidence of NHIS registered PCa increased about threefold from 4,425 in 2006 to 15,043 in 2016 (Table 2). In 2016, the most prevalent PCa district was Jeollanam-do, which was Seoul in 2006 (Fig. 3). In every year, the incidence of PCa was the highest in a rural area (Fig. 4), where had the oldest mean age (Table 3).

\section{DISCUSSION}

The ultimate aim of a population-based screening strategy is to promote cancer-specific survival. About the benefit from the mass screening policy based on PSA, however, there is still controversy particularly since 2012, ${ }^{6,7}$ when the US Preventive Study Task Force (USPSTF) recommended the ban of PSA screening for the mass population mainly due to the lack of positive evidence on the prolonging the survival by applying it to the screening, rather generating overdiagnosis and overtreatment on so-called an insignificant disease. ${ }^{8}$ Even though USPSTF modified their original recommendations in 2018, opening slots of PSA screening for the explained individuals aged between 55 to 69 years, the macroscopic consequence of accepting a specific screening strategy can be quite different in each country, based on the exposure rate to the PSA testing and manifestation of racial differences in PCa incidence. In this point, how the contemporary Western guidelines on PSA screening that are strongly affected by the statements from the USPSTF apply to Korean males' remains unclear, when one considers the lack of data on the frequency of PSA testing and distinctive difference in the proportion of the aged population among the newly diagnosed PCa in Korea.

Nowadays, the majority of PCa was found in the non-metastatic stage, mainly due to the enhanced

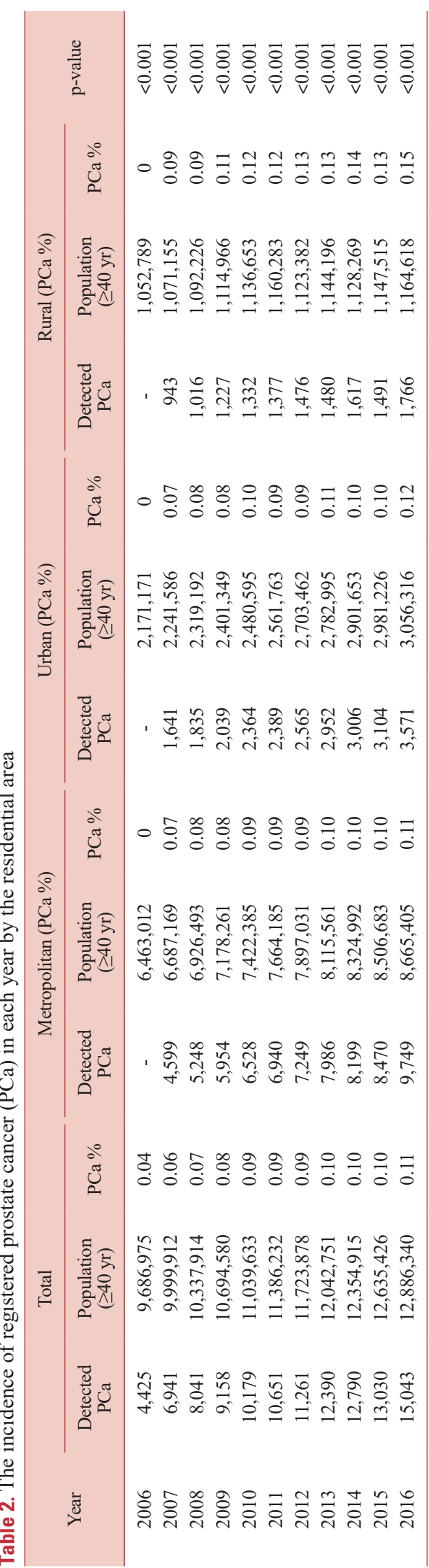




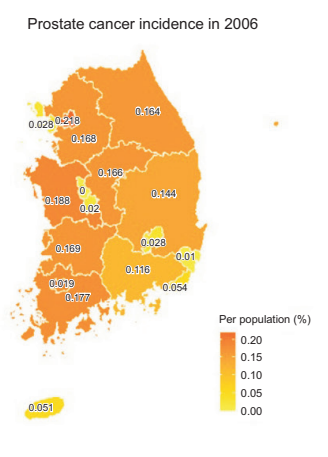

Prostate cancer incidence in 2010

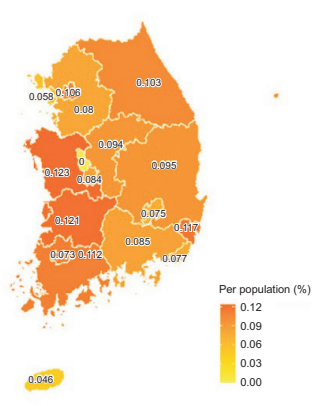

Prostate cancer incidence in 2014

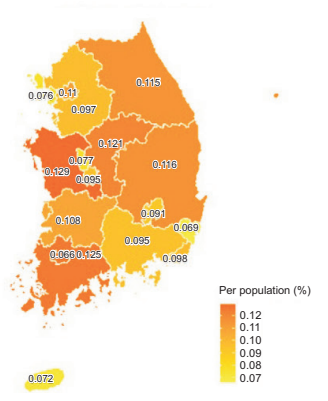

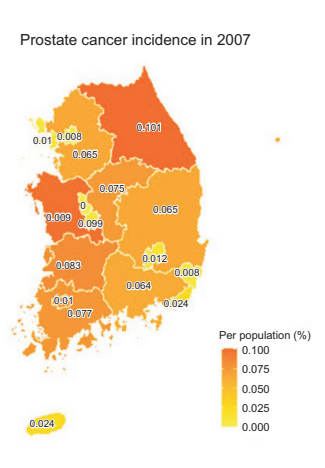

Prostate cancer incidence in 2011

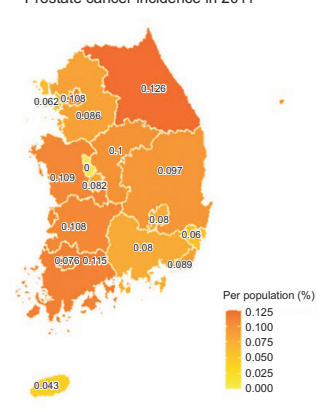

Prostate cancer incidence in 2015

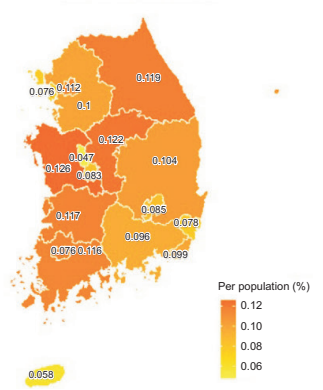

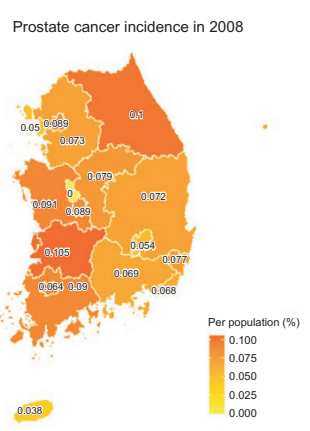

Prostate cancer incidence in 2012
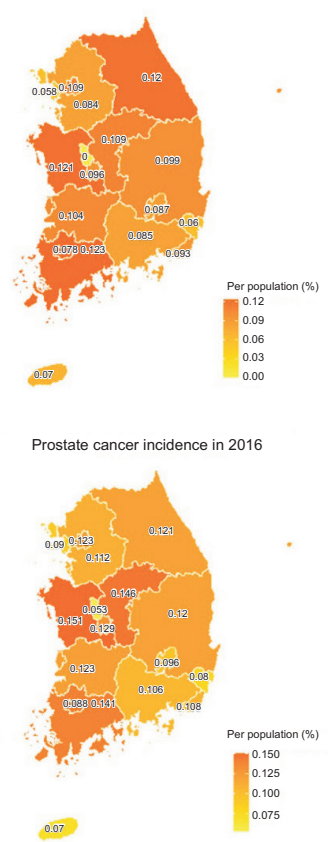

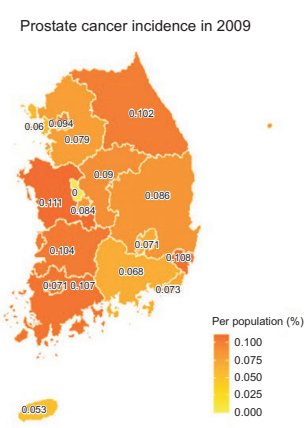

Prostate cancer incidence in 2013

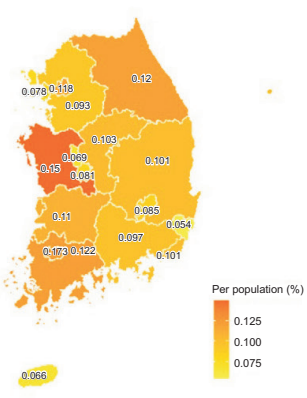

Fig. 3. The incidence of registered prostate cancer in each year by the 17 administrative districts.

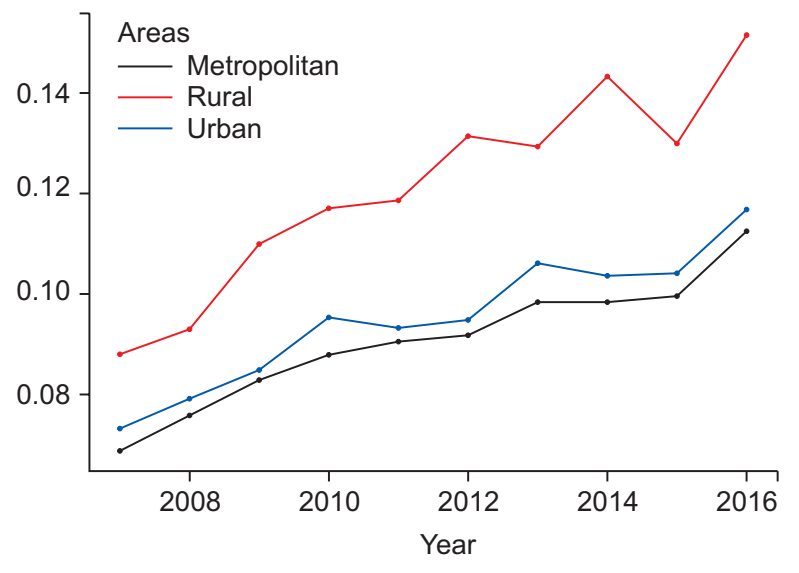

Fig. 4. The incidence of prostate cancer in each year by the residential area. public awareness of the prostatic disease that often develops in the aging male, along with the increased detection by PSA testing. As a result, the incidence of PCa in Korea increased dramatically, more than 12 times just within a couple of decades by the most updated national-wide cancer registry (1,258 in 2000 vs. 14,857 in 2018), even in the absence of a regular check-up policy on PCa in contrast to other prevalent malignant diseases such as lung, stomach, colon, and liver cancer, that currently is the $1 \mathrm{st}, 2 \mathrm{nd}$, and 5 th prevalent male cancer in Korea. ${ }^{4}$ As with other Asian countries, the peculiar characteristic in the epidemiologic feature of PCa is that it primarily encroaches on 


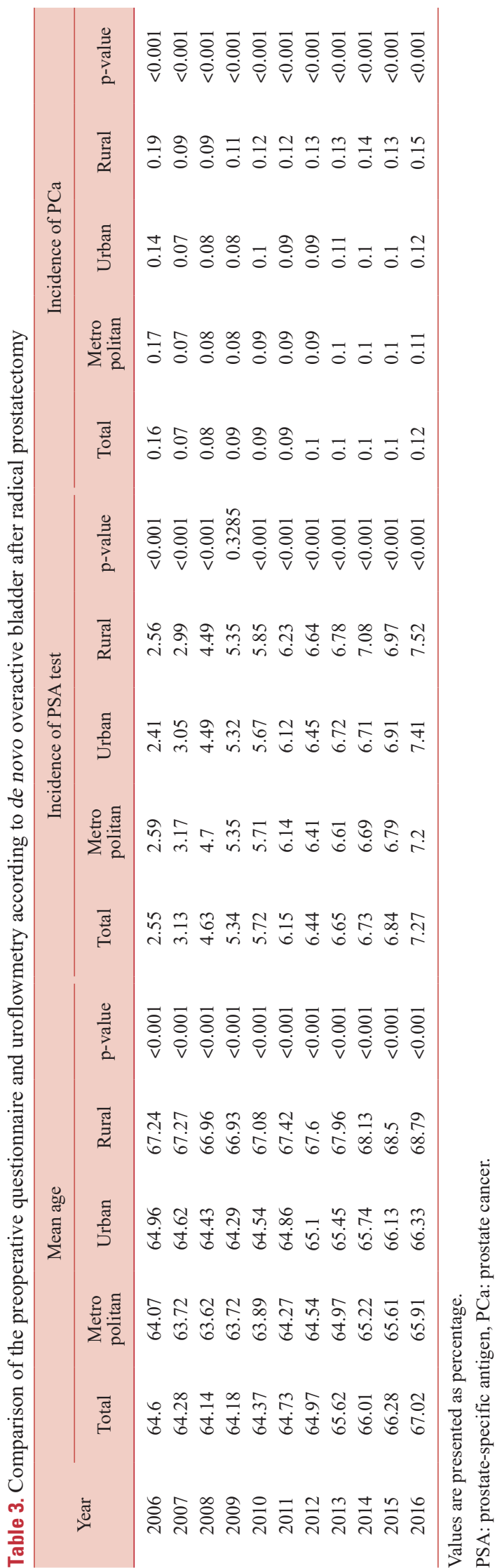

the elderly with age-related increasing incidence rates. $^{1,2}$ Therefore, considering the prominent trend of social again in the rural area than urban or metropolitan counterparts, we are going to contrast the incidence of PSA across these residential areas from this study.

Several interesting findings that deserve to be emphasized were observed from this study. First, the investigated PSA incidence of $7.27 \%$ among those aged over 40 years from this study shown in Table 1 was significantly lower than that from other countries. In a PLCO (Prostate, Lung, Colorectal, and Ovarian Cancer Screening Trial) study carried out in the US, they reported that over $90 \%$ of men in the 'usual care' arm underwent some degree of PSA testing in 2009. ${ }^{6}$ In 2005, it has been reported that about $40 \%$ of those aged over 50 years in the US had at least one PSA test in the prior year. ${ }^{10} \mathrm{~A}$ Japanese hospital-based cohort study reported that $73.5 \%$ of $\mathrm{PCa}$ patients aged $55-69$ years were detected by PSA screening in 2014, ${ }^{11}$ but in Korea, about a quarter of diagnosed PCa had repeated PSA test before the diagnosis in the recent decade. ${ }^{12}$

Second, despite a similarly increasing exposure rate to the PSA test across all residential areas among the Korean male, the repeated screening opportunity was focused on the inhabitant of a metropolitan area. The value of a single session of PSA test as a screening modality has been investigated by several recent trials, but they consistently failed to demonstrate a similar clinical relevance as serial check-ups had. ${ }^{13,14}$ Thus, the ideal screening strategy has to be designed to provide serial check-ups by repeated PSA tests. From this point of view, the relatively lower interval of repeated test of rural area (2.83 times on average during 11 years) in comparison to the inhabitant of a metropolitan area (3.25 times in Seoul, 2.97 times on average from metropolitan area) deserves to be addressed. Given the maintained trend of the highest incidence of PCa in a rural area than 
the other areas, this disparity by the residential area should be reconsidered from the viewpoint of equality, in the accessibility of medical services.

The authors are well aware of the limitations of this study. First, the current version of the Korean NHIS data does not provide basic information on risk stratification of $\mathrm{PCa}$, including the serum level of PSA, clinical stage, and Gleason score or grade. Thus, we cannot identify the significant disease among the indolent PCa in this analysis. While the majority of randomized clinical trials on the efficacy of the PSA test as a screening tool for PCa demonstrated a significant decrease in the incidence of advanced disease, which reflects the probability of low-risk PCa dominantly screened by this strategy. ${ }^{15}$ However, concerns over the overdiagnosis and overtreatment of insignificant PCa should be weighted with the concerns on consistently reported aggressive nature of disease among the Korean males. ${ }^{16-18}$ Second, because the current version of NHIS did not allow to capture the patients who previously had PSA testing performed through a private, noninsured health checkup, some of the nonscreened groups in this study may have proper PSA screening in the real world. The number of those with PSA testing by the private sector was unknown due to the absence of a national-wide counting system. However, given the current reported average retirement age of 51.2 years from their longest employment among the Korean male in $2021^{19}$ and the elderly-driven prevalence PCa in Korea as shown in Table 3, the omission of these private PSA testing data is unlikely to have substantially affected our results, especially for the inhalant of rural area. Third, although associations between the residential area, the mean age, the incidence of PSA and PCa were identified from this study, a causal relationship was not clear from the observational study design. Furthermore, the variables which allow being investigated by the current version of NHIS look superficial to extract a solid conclusion. Thus, these results should be considered as hypothesisgenerating and will spur further investigations into PSA screening in societies with limited social awareness of the disease.

\section{CONCLUSIONS}

Despite a recent increase of PSA screening and diagnosis of $\mathrm{PCa}$ especially in the rural area driven by a larger proportion of the aged population, the opportunity of repeated testing was presented more frequently to the inhabitant of a metropolitan area. Given the relatively low exposure rate to the PSA test regardless of the residential areas in general and the uneven nature of PSA testing by the lack of screening, these outcomes propose the clinical relevance for the establishment of countrywide policy which enables expanding the implementation of PSA testing, in a country with rapidly growing PCa incidence.

\section{NOTES}

- Conflicts of interest: No potential conflict of interest relevant to this article was reported.

- Funding: This work was supported by The Hannam Urological Association (2020).

- ORCID

Young Hwii Ko: https://orcid.org/0000-0002-9150-4292

Byung Hoon Kim: https://orcid.org/0000-0001-5272-6362

\section{REFERENCES}

1. Jemal A, Bray F, Center MM, Ferlay J, Ward E, Forman D. Global cancer statistics. CA Cancer J Clin 2011;61:69-90.

2. Rice KR, Colombo ML, Wingate J, Chen Y, Cullen J, McLeod DG, et al. Low risk prostate cancer in men $\geq 70$ years old: to treat or not to treat. Urol Oncol 2013;31:755-60. 
3. Ito K, Oki R, Sekine Y, Arai S, Miyazawa Y, Shibata $Y$, et al. Screening for prostate cancer: History, evidence, controversies and future perspectives toward individualized screening. Int J Urol 2019;26: 956-70.

4. National Cancer Information Center. Annual report of cancer statistics in Korea in 2019 [Internet]. Goyang (Korea): National Cancer Information Center; 2021 Dec 30 [cited 2022 Jan 3]. Available from: https://ncc.re.kr/cancerStatsView.ncc.

5. Hur HW, Ryu SY, Park J, Choi SW. Relationship between socioeconomic status and prevalent prostate cancer in the South Korea. Asian Pac J Cancer Prev 2019;20:3137-44.

6. Schröder FH, Hugosson J, Roobol MJ, Tammela TL, Ciatto S, Nelen V, et al. Screening and prostatecancer mortality in a randomized European study. N Engl J Med 2009;360:1320-8.

7. Grossman DC, Curry SJ, Owens DK, Bibbins-Domingo K, Caughey AB, Davidson KW, et al. Screening for prostate cancer: US Preventive Services Task Force Recommendation Statement. JAMA 2018;319: 1901-13.

8. Moyer VA, U.S. Preventive Services Task Force. Screening for prostate cancer: U.S. Preventive Services Task Force recommendation statement. Ann Intern Med 2012;157:120-34.

9. US Preventive Services Task Force, Grossman DC, Curry SJ, Owens DK, Bibbins-Domingo K, Caughey $\mathrm{AB}$, et al. Screening for prostate cancer: US Preventive Services Task Force Recommendation Statement. JAMA 2018;319:1901-13.

10. Negoita S, Feuer EJ, Mariotto A, Cronin KA, Petkov VI, Hussey SK, et al. Annual report to the nation on the status of cancer, part II: recent changes in prostate cancer trends and disease characteristics. Cancer 2018;124:2801-14.

11. Kitagawa Y, Mizokami A, Namiki M. Trends of clinical symp-toms and prognosis of middle-aged prostate cancer patients after instigation of pros- tate specific antigen-based population screening. Prostate Int 2013;1:65-8.

12. Ko YH, Kim SW Influence of repeated prostatespecific antigen screening on treatment pattern in a country with a limited social perception of prostate cancer: Korean national wide observational study. Investig Clin Urol 2021;62:282-9.

13. Martin RM, Donovan JL, Turner EL, Metcalfe C, Young GJ, Walsh EI, et al. Effect of a low-intensity PSA-based screening intervention on prostate cancer mortality: the CAP random-ized clinical trial. JAMA 2018;319:883-95.

14. Park J, Suh B, Shin DW, Hong JH, Ahn H. Changing patterns of primary treatment in Korean men with prostate cancer over 10 years: a nationwide population based study. Cancer Res Treat 2016;48:899906.

15. Ilic D, Djulbegovic M, Jung JH, Hwang EC, Zhou Q, Cleves A, et al. Prostate cancer screening with prostate-specific an-tigen (PSA) test: a systematic review and meta-analysis. BMJ 2018;362:k3519.

16. Song C, Ro JY, Lee MS, Hong SJ, Chung BH, Choi HY, et al. Prostate cancer in Korean men exhibits poor differentiation and is adversely related to prognosis after radical prostatectomy. Urology 2006;68:820-4.

17. Kang DI, Chung JI, Ha HK, Min K, Yoon J, Kim W, et al. Korean prostate cancer patients have worse disease characteristics than their American counterparts. Asian Pac J Cancer Prev 2013;14:6913-7.

18. Jeong IG, Dajani D, Verghese M, Hwang J, Cho YM, Hong JH, et al. Differences in the aggressiveness of prostate cancer among Korean, Caucasian, and African American men: a retrospective cohort study of radical prostatectomy. Urol Oncol 2016;34:3.e914.

19. Statistics Korea. The economically active population survey [Internet]. Daejeon (Korea): Statistics Korea; 2021 July 27 [cited 2022 Jan 3]. Available from: http://kosis.kr. 